\title{
New potentials of mitochondrial DNA editing
}

\author{
Willian Wang • Xiangdong Wang
}

Received: 15 July 2020 / Accepted: 21 July 2020/Published online: 30 July 2020

(C) Springer Nature B.V. 2020

\begin{abstract}
Gene modification and editing using mitochondrial DNA (mtDNA) is a promising option to conventional therapy for mitochondrial dysfunction which can result in a wide range of diseases and failure of organ and tissue functions. RNA-free DddA-derived cytosine base editors have been a promising method due to its high target specificity and high product purity in human mtDNA. However, mtDNA editing is still only a promising option and further studies and investigations are required prior to implementing it in different cell types, organelles, and various diseases as a new clinical therapy.
\end{abstract}

Keywords mtDNA $\cdot$ Genes $\cdot$ Editing $\cdot$ Mitochondria

Mitochondria play important roles in the maintenance of organ/tissue function in the body by mitochondrial genetic messaging, energy production, and metabolism. Mitochondrial dysfunction is responsible for the development of cell metabolism disorders, tissue edema and injury, diseases, cell resistance against drugs, and genetic heterogeneity (Fang et al. 2019; Gutierrez et al. 2019; Hasnat et al. 2019). Of those mitochondrial elements, mitochondrial DNA (mtDNA) is crucial in the communication with nuclear messages, organelles, and cells, as well as regulating cell response, autophagy/mitophagy, genome activation, and cell interaction (Wu et al. 2018). mtDNA is a

W. Wang $\cdot$ X. Wang $(\bowtie)$

Zhongshan Hospital Institute of Clinical Science, Fudan University Shanghai Medical College, Shanghai, China e-mail: xdwang@fuccb.com control center for mitochondrial-epigenetic crosstalk, the occurrence of mitophagy, desensitization of targeted therapy, and dysfunction of mitochondrial respiratory megacomplexes. The stability of mtDNA is maintained and regulated by methylation of mtDNA, mtDNA methylation-associated networks and factors, methyltransferases, nucleotides, mt-rRNAs, and other epigenetic modifications. Defining mitochondrial functions was considered to be a milestone, and developing a better understanding of how genome DNA and mtDNA interacts, their intracellular signaling pathways, patient phenomes, and mtDNA is crucial in developing methods for DNA editing (Wang et al. 2020a). Functional profiles and regulations of mtDNA methylation are dependent upon the number of $\mathrm{s}$ control region sites for methylation, which enzymes and regulators are involved, and the differentiation among cell types, species, functions, and diseases. In addition, targeted gene editing of mtDNA can provide new insights for understanding novel functions of mtDNA and for developing new therapies for human maternally inherent mitochondrial diseases or other diseases. The aims of this editorial are to briefly overview the recent development of mtDNA editing/modification and to call special attention to exploring new potential venues for mtDNA therapy.

Mitochondrial heteroplasmy is a factor influencing the stability and function of mtDNA by altering the copy number, sequencing disorder, and point deletion which are responsible for inherited diseases. mtDNA editing/ modification was initiated to cure heteroplasmyassociated mitochondrial dysfunction and diseases and has now been considered further as potential treatment for heteroplasmy-dependent or independent diseases 
(Sun et al. 2017). In addition to the challenges facing heteroplasmy diagnosis due to the great variation among cells, tissues/organs, and diseases, it is more difficult to develop and define the methodologies of mtDNA editing/ modification. For example, about $95 \%$ of patients with mitochondria-dependent Leber hereditary optic neuropathy (LHON) have point mutations in three mitochondrial genes for structural subunits of complex I account; other mutations also exist in MT-ND1, MT-ND4, MT-ND6, MT-ND2, MT-ND4L, MT-ND5, MT-ATP6, MT-CO3, and MT-CYB (Prasun n.d.). The difficulties mtDNA editing needs to address before curing LHON include developing a method to precisely target mutations due to heteroplasmy and incomplete penetrance, designing therapeutic strategies for individuals due to variation between males and females, defining mitochondrial haplogroup with more harmful mutations, and preventing reoccurrence of diseases due to increased penetrance of LHON mutation by multiple environmental factors. Preclinical and clinical efforts have been made to treat and prevent the occurrence of mitochondrial diseases by gene editing in germline cells and embryos, mitochondrial implantations, RNA editing, and DNA editing. The methods targeting pathogenic mtDNA for heteroplasmy shift have been improved and developed for antigenomic therapy by genome engineering and modification, including meganucleases, zinc finger nucleases (ZFNs), transcription activator-like effector nuclease (TALENs), clustered regularly interspaced short palindromic repeats (CRISPR) systems, and several novel genome editing tools such as base editors and prime editing, although there are still many considerations on off-target effects (Zheng et al. 2020).

With the rapid development of gene editing, mtDNA modifications with fewer side effects can provide a new pathway to developing target-oriented molecular networks, interactions, and mitochondrial biology. It can also help to develop target-based therapy for mitochondrial mutation-specific diseases. Gammage et al. treated the m.5024C $>$ T tRNAAla mouse with molecular phenomes of the heteroplasmic mtDNA disease by systemically administering the virus delivery system with ZENs and found that those ZENs targeted to cardiac tissue mitochondria and eliminated specific mutations of mtDNA (Gammage et al. 2018). Such a programmable nuclease therapy could improve molecular and biochemical phenotypes of heteroplasmic mitochondrial diseases. Redy et al. demonstrated that targeted eliminations of mutated mtDNA could correct the heteroplasmy shift using mitochondria-targeted restriction endonucleases or TALENs, to prevent germline transmission of mitochondrial diseases (Reddy et al. 2015). Target mtDNA gene editing reduced human mutated mtDNA levels responsible for LHOND and clinical phenomes (e.g., neurogenic muscle weakness, ataxia, and retinitis pigmentosa). Bian et al. applied a mitochondrial CRISPR/Cas9 system to specifically target mtDNA, reduce mtDNA copy number, and knock an exogenous single-stranded DNA with short homologous arm in human cells and zebrafish (Bian et al. 2019). More recently, Mok et al. reported an interbacterial cytidine deaminase toxin (DddA) enabling CRISPR-free mitochondrial base editing to catalyze the deamination of cytidine in double-strand DNA (dsDNA) as a CRISPR-free, RNA-free base editor to modify targeted mutations in the human mitochondrial genome (Mok et al. 2020). This is one of gene editing milestones, to convert $\mathrm{C} \cdot \mathrm{G}$-to-T•A in mtDNA without double-strand breaks, offering the potential to create mitochondrial disease mutation models, and to rearrange pathogenic sequences for therapy, although further validations are needed in more cell and animal disease models. This RNA-free DddA-derived cytosine base editors (DdCBEs) have high target specificity and product purity in human mtDNA, which could be applied in other intracellular organelles beyond mitochondria, such as in different cell types, various animals, and the possibility of therapeutic applications. For clinical application, it is questioned whether DdCBEs has the specificity targeting pathogenic variants in organelles from the circulation after systemic administration. Other questions such as the long-term off-target effects and toxicity, the consequences of non-specific binding of transcription activator-like effectors, and the therapeutic efficacy to improve clinical phenomes remain and will need to be addressed prior to clinical application.

In addition to mitochondrial genetic diseases, mtDNA mutations and mtDNA-dependent mitochondrial heteroplasmy appear in many pathological conditions and are responsible for disease pathogenesis, development, and prognosis. For example, somatic mutations of mtDNA often occurred in cancer cells to meet the alterations in metabolism for progression, metastasis, and drug response (Guerra et al. 2017). mtDNAassociated mitochondrial dysfunction also played an important role in the pathophysiological processes of chronic lung diseases (Fang et al. 2019), e.g., chronic obstructive pulmonary disease, chronic lung fibrosis, or 
chronic asthma. Mitochondrial fusion and fission as well as lipid metabolisms can vary with the dynamic changes of mtDNA mutations (Zeng et al. 2020). mtDNA mutations may promote the bioenergetic and biosynthetic profiles of cancer cells, inflammatory cells, and tissue structure cells through signaling interactions between the nucleus and mitochondria, other organelles and mitochondria, and mitochondria and mitochondria (Guerra et al. 2017; Wang et al. 2020b; Zeng et al. 2020; Wang et al. 2020c). For example, deoxynucleoside triphosphate (dNTP) pools act as a bridge between nuclear and mitochondrial genome replication and repair, during which imbalanced mitochondrial dNTP pools can cause the occurrence of mtDNA mutations through target enzyme deficiency and inactivation in dNTP synthesis, mtDNA replication, and/or other metabolic pathways. On the other hand, mtDNA mutations also influence de novo synthesis and activity of dNTP pool-associated enzymes and the separation of dNTP pools. Thus, it would be more valuable if mtDNA gene editing can be of benefit for multi-gene mutations, mitochondrial dysfunction-associated diseases, and intratumor and inflammatory metabolic heterogeneity. mtDNA gene editing may affect dNTP and mitochondrial nucleotide synthesis, purine and pyrimidine metabolism, and mitochondrial nucleotide metabolism with amino acids, glucose, and fatty acid metabolism.

In conclusion, mtDNA gene editing/modification can be an alternative to conventional therapy for mitochondrial dysfunction which plays an important role in the development of various diseases. Among the many approaches to mtDNA editing, RNA-free DddA-derived cytosine base editors are considered to have high target specificity with high product purity in human mtDNA. Further investigations are needed prior to mtDNA editing for other intracellular organelles, cell types, and diseases as a new therapy.

\section{References}

Bian WP, Chen YL, Luo JJ, Wang C, Xie SL, Pei DS. Knock-in strategy for editing human and zebrafish mitochondrial DNA using mito-CRISPR/Cas9 system. ACS Synth Biol. 2019;8(4):621-32. https://doi.org/10.1021/acssynbio.8 b00411.

Fang T, Wang M, Xiao H, Wei X. Mitochondrial dysfunction and chronic lung disease. Cell Biol Toxicol. 2019;35(6):493502. https://doi.org/10.1007/s10565-019-09473-9.
Gammage PA, Viscomi C, Simard ML, Costa ASH, Gaude E, Powell CA, et al. Genome editing in mitochondria corrects a pathogenic mtDNA mutation in vivo. Nat Med. 2018;24(11): 1691-5. https://doi.org/10.1038/s41591-018-0165-9.

Guerra F, Arbini AA, Moro L. Mitochondria and cancer chemoresistance. Biochim Biophys Acta Bioenerg. 2017;1858(8):686-99. https://doi.org/10.1016/j. bbabio.2017.01.012.

Gutierrez DA, DeJesus RE, Contreras L, Rodriguez-Palomares IA, Villanueva PJ, Balderrama KS, et al. A new pyridazinone exhibits potent cytotoxicity on human cancer cells via apoptosis and poly-ubiquitinated protein accumulation. Cell Biol Toxicol. 2019;35(6):503-19. https://doi.org/10.1007 /s10565-019-09466-8.

Hasnat M, Yuan Z, Naveed M, Khan A, Raza F, Xu D, et al. Drp1associated mitochondrial dysfunction and mitochondrial autophagy: a novel mechanism in triptolide-induced hepatotoxicity. Cell Biol Toxicol. 2019;35(3):267-80. https://doi. org/10.1007/s10565-018-9447-8.

Mok BY, de Moraes MH, Zeng J, Bosch DE, Kotrys AV, Raguram $\mathrm{A}$, et al. A bacterial cytidine deaminase toxin enables CRISPRfree mitochondrial base editing. Nature. 2020;583:631-7. https://doi.org/10.1038/s41586-020-2477-4.

Prasun P. Mitochondrial medicine: a primer for health care providers and translational researchers. New York: Elsevier. 2019. https://doi.org/10.1016/C2018-0-01187-1.

Reddy P, Ocampo A, Suzuki K, Luo J, Bacman SR, Williams SL, et al. Selective elimination of mitochondrial mutations in the germline by genome editing. Cell. 2015;161(3):459-69. https://doi.org/10.1016/j.cell.2015.03.051.

Sun H, Shi W, Wang X. How far can mitochondrial DNA drive the disease? Adv Exp Med Biol. 2017;1038:1-8. https://doi. org/10.1007/978-981-10-6674-0_1.

Wang W, Liu X, Wang X. How to breakthrough mitochondrial DNA methylation-associated networks. Cell Biol Toxicol. 2020a;36(3):195-8. https://doi.org/10.1007/s10565-02009539-z.

Wang W, Li L, Wang X. Therapeutic targets during mitochondrial lipid metabolism. Cell Biol Toxicol. 2020b;36(3):205-8. https://doi.org/10.1007/s10565-020-09543-3.

Wang Y, Liu X, Shi H, Yu Y, Yu Y, Li M, et al. NLRP3 inflammasome, an immune-inflammatory target in pathogenesis and treatment of cardiovascular diseases. Clin Trans1 Med. 2020c;10(1):91-106. https://doi.org/10.1002/ctm2.13.

Wu D, Wang X, Sun $\mathrm{H}$. The role of mitochondria in cellular toxicity as a potential drug target. Cell Biol Toxicol. 2018;34(2):87-91. https://doi.org/10.1007/s10565-018-9425-1.

Zeng B, Huang Y, Jin S, Zhang X, Zhang H, Shi G, et al. Development and validation of a mitochondrial metabolism-associated nomogram for prediction of prognosis in clear cell renal cell carcinoma. Clin Transl Med. 2020. https://doi.org/10.1002/ctm2.120.

Zheng N, Li L, Wang X. Molecular mechanisms, off-target activities, and clinical potentials of genome editing systems. Clin Transl Med. 2020;10(1):412-26. https://doi.org/10.1002 /ctm2.34.

Publisher's note Springer Nature remains neutral with regard to jurisdictional claims in published maps and institutional affiliations. 